\title{
Photonic-crystal resonant effect using self-assembly ordered structures in magnetic fluid films under external magnetic fields
}

\author{
S.Y. Yang, H.E. Horng ${ }^{\mathrm{a}, *, 1}$, Y.T. Shiao ${ }^{\mathrm{a}}$, Chin-Yih Hong ${ }^{\mathrm{b}, * *}$, H.C. Yang ${ }^{\mathrm{c}}$ \\ ${ }^{a}$ Institute of Electro-optical Science and Technology, National Taiwan Normal University, Taipei 116, Taiwan \\ ${ }^{\mathrm{b}}$ Department of Mechanical and Automation Engineering, Da-Yeh University, Changhwa 515, Taiwan \\ ${ }^{\mathrm{c}}$ Department of Physics, National Taiwan University, Taipei 106, Taiwan
}

Received 31 December 2005; received in revised form 24 February 2006

Available online 17 April 2006

\begin{abstract}
When an external magnetic field is applied perpendicularly to a magnetic fluid film, a self-assembly hexagonal ordered structure is formed in the film when the field exceeds a certain strength. The period of the ordered structure can be manipulated from several micrometers down to sub-micrometers by increasing the field strength or reducing the film thickness. By injecting a light propagating along the in-plane direction of the film, photonic-crystal band gaps can be observed. With the tunability of the ordered structure, the forbidden photonic bands can be adjusted by varying the external field strength. It was further found that resonant modes are generated when a point defect is artificially implanted into the self-assembly ordered structure in the magnetic fluid film. These results reveal that the self-assembly ordered structure in a magnetic fluid film under external magnetic fields acts as a photonic crystal.
\end{abstract}

(C) 2006 Elsevier B.V. All rights reserved.

PACS: 42.70.Qs; 85.70.Sq; 75.50.Mm

Keywords: Photonic crystals; Magnetic fluids; Resonant modes

\section{Introduction}

Due to the spatially periodic variation in the refractive index, photonic crystals exhibit spectacular diffractions for incident lights, with a number of new phenomena having been reported since photonic crystals were discovered in 1987 [1,2]. At the present time, scientists and engineers have been focusing their efforts on defect-mode engineering $[3,4]$, band-gap-size engineering [5,6], and gap-edge-mode engineering $[7,8]$. In addition to exploring the physical mechanisms for these engineering fields, many nanophotonic devices have been developed, such as polarizer, cavities [3,4], waveguides [9], couplers [10], superlens [11], superprism [12], and so on. Such advances, made capable

\footnotetext{
*Corresponding author. Tel.: + 886229336260 ; fax: +886286631954 .

**Also to be corresponded to. Tel.:/fax: +88648511213 .

E-mail addresses: phyfv001@scc.ntnu.edu.tw (H.E. Horng), cyhong@mail.dyu.edu.tw (C.-Y. Hong).

${ }^{1}$ Also with Department of Physics, National Taiwan Normal University, Taipei 116, Taiwan.
}

by photonic crystals, have had great impacts on optical communication, optical storage, laser engineering, biosensor technology, etc. Therefore, photonic crystals have continually attracted the interest of researchers.

The most popular technology used to fabricate photonic crystals is the e-beam lithography [13,14]. Other techniques such as self-assembling [15], direct writing [16], multi-beam interference [17], auto-cloning [18], etc. are also utilized for making photonic crystals. In this work, another method, which invokes the self-assembling ordered structures in magnetic fluid films, is exploited to achieve two-dimensional photonic crystals.

Magnetic fluids are a kind of colloids that possess magnetic nano-particles dispersed in liquid with an aid of surfactant on the particles and through Brownian motion. Each particle is magnetically single domain. When an external magnetic field is applied to a magnetic fluid film, the particles agglomerate due to the attraction among particles, and magnetic clusters are formed. These magnetic clusters form rich structural patterns. The studies on the 
magnetically induced instability of magnetic fluid films were started up in 1980s by pioneers like Khizhenkov $[19,20]$, Tsebers [21,22], and so on. From then on, the pattern formation of magnetic fluid films under magnetic fields received increasing studies [23-28]. For example, under magnetic fields perpendicular to magnetic fluid films, the structural pattern may evolve from a disordered distributed columns to ordered structure, and then to a labyrinthine pattern [27]. Besides, many reports reveal the fringing features of concentrated micro-drops in magnetic fluid films under external magnetic fields $[22,28]$. With the success in synthesizing highly homogeneous magnetic fluids [29], Horng et al. [30] found an ordered array of magnetic clusters in a magnetic fluid film under external magnetic fields. Owing to the ordered structure in the magnetic fluid film, the refractive index varies periodically over the film. Hence, diffraction occurs when lights are incident normally to the film. In this work, we investigate the photonic crystal effect of the self-assembling ordered structure in a magnetic fluid film when the lights are incident along the in-plane direction. Furthermore, a photonic crystal cavity is designed and demonstrated that utilizes the ordered structure in the magnetic fluid films.

\section{Experiment and simulation}

The magnetic fluid used in this work was kerosene-based ferrofluid possessing magnetic nano-particles of $\mathrm{MnFe}_{2} \mathrm{O}_{4}$. The chemical synthesis of magnetite $\mathrm{MnFe}_{2} \mathrm{O}_{4}$ by coprecipitation of $\mathrm{MnSO}_{4}$ and $\mathrm{FeCl}_{3}$ in the presence of $\mathrm{NaOH}$ is based on a reaction proposed. This co-precipitation reaction has been used to generate ferrofluids in which the $\mathrm{MnFe}_{2} \mathrm{O}_{4}$ magnetic nano-particles are coated with oleic acid surfactant and dispersed in kerosene carrier. The details about the synthesis of ferrofluid used here are given in our previous report [30]. By using Microtrac Nanotrac 150 based on dynamic laser scattering, in which the particle size was measured by detecting the Brownian motion of the particles through probing the Doppler frequency shift of a scattered light with respect to the incident light, the averaged diameter of magnetic nanoparticles was found to be $17.2 \mathrm{~nm}$ with a standard deviation of $3.2 \mathrm{~nm}$.

To form a magnetic fluid film, the ferrofluid was injected into a hexagonal-shaped silicon cell, shown in Fig. 1(a), and then covered with a glass plate. A silicon rod may be fabricated at the center of the cell to act as a point defect for the magnetically induced ordered structure in the magnetic fluid film. The silicon point defect is fabricated as a cylindrical rod with a diameter of $1 \mu \mathrm{m}$ here. An optical microscope and a CCD camera were used to record the structure in the magnetic fluid film. The magnification of the microscope (Olympus BH2-UHA) is 1000 with an aid of a long-working-distance object (x100), and the spatial resolution is about $0.55 \mu \mathrm{m}$.

The theoretical photonic band structure of an ordered structure can be simulated by solving the master

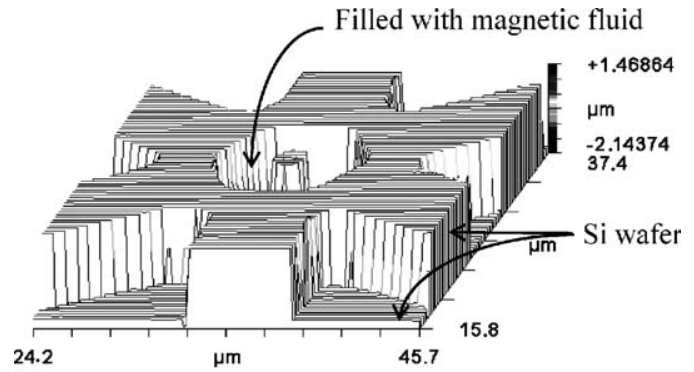

(a)

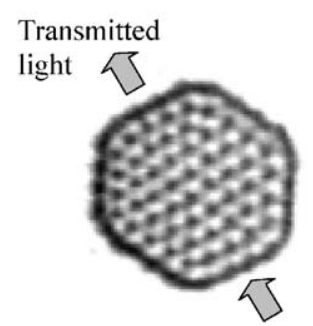

(b) Incident light

(c)

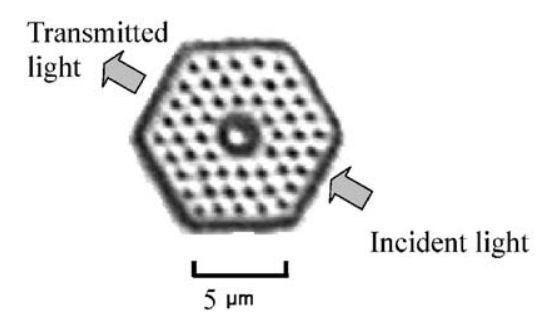

Fig. 1. (a) Three-dimensional image of the silicon cell to contain magnetic fluid. Images of ordered structures in the magnetic fluid film (b) without (c) with a silicon rod at the center. The external magnetic field is applied perpendicularly to the film up to $10 \mathrm{mT}$ from zero at a sweep rate $1 \mathrm{mT} / \mathrm{s}$. The film thickness, i.e. cell depth, is $2.0 \mu \mathrm{m}$, and the concentration of the magnetic fluid is $23.43 \%$.

equation

$\nabla \times \frac{1}{\varepsilon} \nabla \times \vec{H}=\left(\frac{\omega}{c}\right)^{2} \vec{H}$

for the magnetic field $H$ of electromagnetic waves propagating in the photonic crystal via the frequencydomain plane-wave expansion method $[31,32]$, where $\varepsilon$ is the dielectric constant and $\omega$ is the frequency of a propagating mode. From the simulated photonic band structures, the forbidden transmitted wavelengths through the investigated ordered structure can be found.

To measure the transmission spectrum of the magnetic fluid film, a broad-band light emitted from an ASE light source was guided by a multi-mode optical fiber to be incident into the film along the in-plane direction, as indicated with arrows in Figs. 1(b) and (c). The transmitted light, which was taken at the opposite to the incident side, was guided to an optical spectrum analyzer to measure the transmission spectrum. The magnetic field, which is required for the formation of ordered structures in the magnetic fluid film, was generated with a solenoid. By using a Gauss meter to measure the spatial variation of the applied magnetic field, it was found that the deviation of 
the applied magnetic field within the sample region is less than $0.02 \%$.

\section{Results and discussion}

When a magnetic field is applied perpendicularly to the magnetic fluid film, magnetic particles which are originally dispersed in kerosene agglomerate to form magnetic columns. If the field strength is increased, more magnetic columns are formed, and then a hexagonal ordered structure is self-assembled in the magnetic fluid film due to the repulsive interactions among magnetic columns. An image of a typical self-assembly hexagonal ordered structure in the magnetic fluid film is shown in Fig. 1(b), in which the external magnetic field is $10 \mathrm{mT}$ applied at a sweep rate of $1 \mathrm{mT} / \mathrm{s}$ from zero and the thickness of the magnetic fluid film is $2.0 \mu \mathrm{m}$. The black dots denote the cross-sections of magnetic columns. The column spacing in Fig. 1(b) is $1.10 \mu \mathrm{m}$, and the column diameter is $0.51 \mu \mathrm{m}$. Thus, the ratio of the column radius to the spacing is 0.23 . Through experience, the column spacing can be tuned from several micrometers down to sub-micrometers by increasing the magnetic field strength $H$, reducing the film thickness $L$, or by applying the field at a higher sweep rate $\mathrm{d} H / \mathrm{d} t$. Fig. 2 gives an image of the ordered structure in a thinner magnetic fluid film of $0.94 \mu \mathrm{m}$ under $20 \mathrm{mT}$ with $\mathrm{d} H / \mathrm{d} t$ being $50 \mathrm{mT} / \mathrm{s}$. The column spacing $d$ is reduced to $0.62 \mu \mathrm{m}$, and the column diameter $a$ becomes $0.33 \mu \mathrm{m}$. As is shown in Fig. 3(a), the column spacing varies from 0.64 to $0.57 \mu \mathrm{m}$ when the field $H$ increases from 15 to $35 \mathrm{mT}$, whereas the column diameter $a$ almost remains the same for a given film under a fixed $\mathrm{d} H / \mathrm{d} t$, as can be seen in Fig. 3(b). It was also observed that the ratio of the column radius to the column spacing $a / 2 d$ is within the range from 0.22 to 0.27 . It is worthy of note that the ordered structure is still formed in the magnetic fluid film under $H$ 's higher than $35 \mathrm{mT}$, but is not shown here because of limitations in the spatial resolution of the microscope and the CCD.

With the ordered structures in the magnetic fluid film under external magnetic fields, the refractive index varies periodically over the film. The forbidden bands may exist in the photonic band structures for the lights propagating

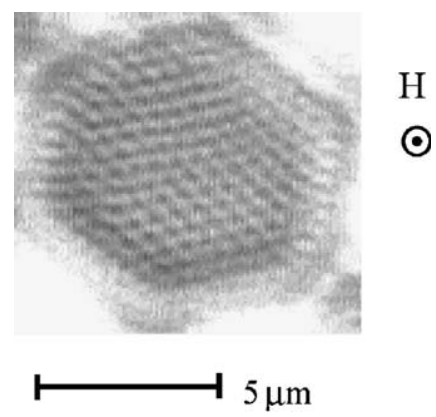

Fig. 2. Ordered structure in a magnetic fluid film of $0.94 \mu \mathrm{m}$ in thickness and $23.43 \%$ in volume concentration. The magnetic field $H$ of $200 \mathrm{Oe}$ is applied at the sweep rate $50 \mathrm{mT} / \mathrm{s}$. The column spacing $d$ is $0.62 \mu \mathrm{m}$ and the column diameter $a$ is $0.33 \mu \mathrm{m}$.

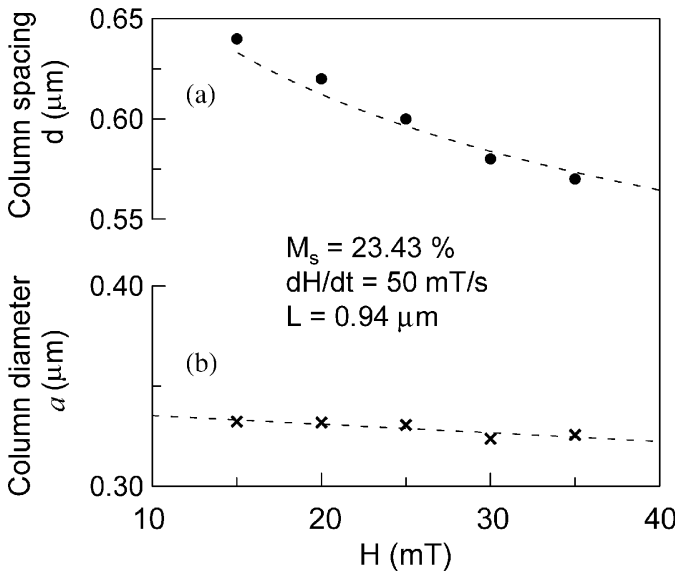

Fig. 3. Field-dependent (a) column spacing $d(H)$ and (b) column diameter $a(H)$ of the ordered structure in a magnetic fluid film. The thickness of the magnetic fluid film is $0.94 \mu \mathrm{m}$, and the concentration of the magnetic fluid is $23.43 \%$. The sweep rate of the applied magnetic field is $50 \mathrm{mT} / \mathrm{s}$.

in the film along the in-plane direction. To simulate the photonic band structures through solving Eq. (1), the geometric factors and the dielectric constant (or refractive indices) of the liquid and the magnetic columns are required. The refractive indices of the liquid and magnetic columns can be evaluated according to measurement results of the refractive index and the transmission of magnetic fluid films under external fields. The detailed estimations on the refractive indices of liquid and columns were discussed in our previous work [33-35]. In brief, a magnetic column can be regarded as a cluster of closestpacking magnetic nano-particles [33]. By taking the contributions form the surfactant (oleic acid) and particles $\left(\mathrm{MnFe}_{2} \mathrm{O}_{4}\right)$ into account, the effective refractive index of a magnetic column is available. It is worthy noting that the refractive index of magnetic columns is independent of the magnetic field strength, whereas the refractive index of liquid depends on the field strength because the particle concentration in liquid varies under various field strengths. Hence, to evaluate the refractive index, we estimate the effective magnetic concentrations of liquid under various magnetic fields first, and then find the effective refractive index of liquid via the empirical relationship between the refractive index and the magnetic concentration of liquid [34]. As a result, the refractive indices of the magnetic column $\left(n_{\text {col }}\right)$ is 3.74 , the 2.3 is obtained for the refractive index of liquid $\left(n_{\text {liq }}\right)$ under $40 \mathrm{mT}$. Thus, the corresponding dielectric constants are 13.99 and 5.29, respectively.

The geometric factors are available from the experimental $d-H$ and $a-H$ curves in Figs. 3(a) and (b) for the magnetic fields up to $35 \mathrm{mT}$. But in order to compare the theoretical forbidden bands with the observed transmission spectra through the ordered structure in the magnetic fluid film under $40 \mathrm{mT}$ shown in Fig. 4(b), the column spacing $d$ and the column diameter $a$ at $40 \mathrm{mT}$ must first be known to calculate the photonic band structures. The values of $d$ and of $a$ at $40 \mathrm{mT}$ can be found as 0.562 and $0.322 \mu \mathrm{m}$, respectively by exploiting the $d-H$ and the $a-H$ curves in 


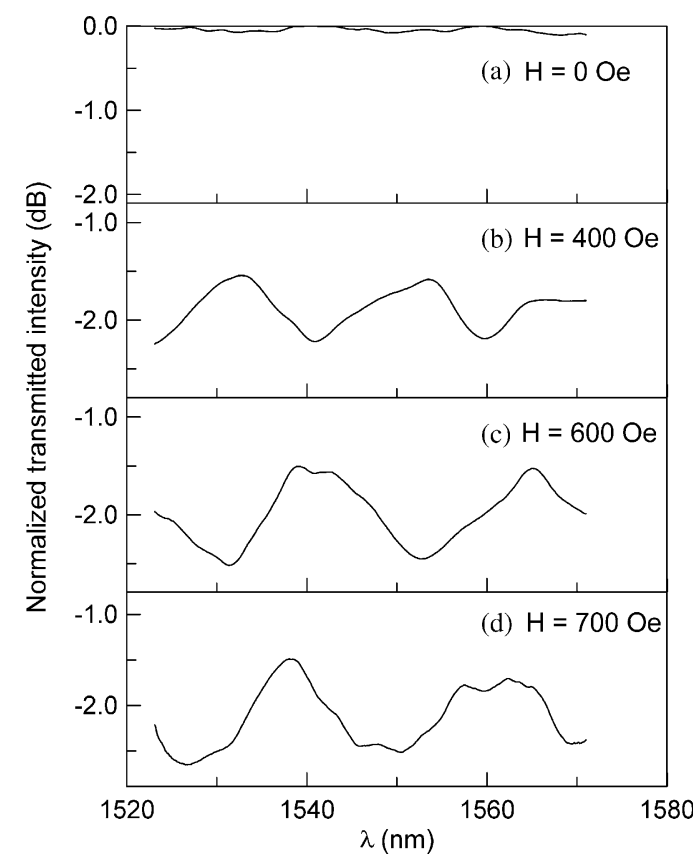

Fig. 4. Transmission spectra of the incident light through th e ordered structures in the magnetic fluid film under various magnetic fields of (a) 0 , (b) 40 , (c) 60 , and (d) $70 \mathrm{mT}$. The scheme of the incident and the transmitted directions of light through the magnetic fluid film is shown in Fig. 1(b). The corresponding field-dependent structural characters of the magnetic fluid film are shown in Figs. 3(a) and (b).

Figs. 3(a) and (b). With the geometric factors and the indices of the ordered structure in the magnetic fluid film under $40 \mathrm{mT}$, the photonic band structures can be simulated. The photonic band structures show that middle normalized frequencies $\omega_{\mathrm{N}}$ ( $=$ the ratio of column spacing to wavelength) of the first and the second forbidden bands are 0.2213 and 0.3602 for TE modes, but no gap occurs for TM modes. Hence, the wavelengths corresponding to the first and the second forbidden bands for TE modes are around 2.5395 and $1.5602 \mu \mathrm{m}$, respectively. Since the wavelength range of the incident lights used here is from 1522 to $1572 \mathrm{~nm}$, the $2.5395 \mu \mathrm{m}$ in-wavelength forbidden band cannot be observed. However, the $1.5602 \mu \mathrm{m}$ inwavelength forbidden band should be observed at $40 \mathrm{mT}$.

Figs. 4(a)-(d) plot the experimental transmission spectra of the magnetic fluid film under various field strengths. In Fig. 4(a), the transmission spectrum through the magnetic fluid film under zero field is shown. The transmission spectrum under $40 \mathrm{mT}$ is given in Fig. 4(b). It is clear that one of the forbidden bands occurs at the wavelength of $1.560 \mu \mathrm{m}$, which coincides with the calculated forbidden wavelength for TE mode. The other two forbidden bands at 1.523 and $1.541 \mu \mathrm{m}$ in wavelength may be due to the unpolarized incident lights used in the experiment. Besides, the low value of $1 \mathrm{~dB}$ for the transmission variation of the forbidden bands may be due to the following reason. Since the six waveguides around the cell are silicon, which is the same material as the substrate, a large portion of incident light into the waveguide will leak out to the silicon substrate and propagate along the silicon substrate instead of the silicon waveguide. The transmission intensity through the silicon substrate does not change regardless if under or without external magnetic fields. Only small portion of the incident light propagating along the waveguide experiences the ordered structure in the magnetic fluid film and may be diffracted. In the experiment, both the transmitted intensities of the lights through the waveguide and the substrate are detected. Hence, the observed variation in the transmitted intensity shown in Fig. 4(b) is depressed due to the large contribution from the transmitted light through the silicon substrate. The leakage of the incident light from the waveguide into the substrate might be possibly prevented by using higher-index material (e.g. $\mathrm{ZnO}$, refractive index $\approx 2.0$ ) as the waveguide and lower-index material (e.g. $\mathrm{SiO}_{2}$, refractive index $\approx 1.4$ ) as the substrate.

In Figs. 4(c) and (d), the forbidden bands are shifted to the regions of short wavelengths under a higher magnetic field. This is attributed to the reduction in the column spacing of the ordered structure in the magnetic fluid film under a higher magnetic field, as evidenced in Fig. 3(a). Thus, the forbidden wavelength can be tuned by tuning the column spacing in the magnetic fluid film via adjusting the magnetic field strength.

It is well known that a resonant wavelength located at a forbidden band is generated when a point defect is implanted into the perfectly ordered structure of a photonic crystal. To study the resonant effect, we artificially created a point defect in the self-assembly ordered structure in the magnetic fluid film. In this work, a silicon rod is fabricated at the center of the film and is used as the point defect. Fig. 1(c) shows the structure of magnetic columns in the magnetic fluid film with a silicon rod at the center under $10 \mathrm{mT}$. It is clear that a hexagonal ordered structure is achieved in the film. The ordered structure in Fig. 1(c) possesses the same column spacing as that of the perfectly ordered structure in Fig. 1(b). Therefore, a structure using magnetic fluid for a photonic crystal cavity is obtained. Then, the in-plane transmission spectrum of the magnetic-fluid ordered structure with a point defect is measured under $60 \mathrm{mT}$. The result is shown in Fig. 5(a) with a dashed line.

In Fig. 5(a), there appear three peaks in the transmission spectrum at wavelengths of 1532,1552 , and $1569 \mathrm{~nm}$, which correspond to the forbidden bands for the perfectly ordered structure plotted with a solid line in Fig. 5(a). Thus, theses three peaks are the resonant wavelengths of the magnetic-fluid photonic crystal with a point defect.

Since the forbidden bands are magnetically manipulated, the resonant wavelengths of the magnetic-fluid ordered structure with a point defect are detected under various magnetic fields from 60 to $70 \mathrm{mT}$ are investigated and are shown in Figs. 5(a)-(c). In Figs. 5(a)-(c), the dashed lines shows the transmission spectra through the magnetic-fluid ordered structure with a point defect under 60,65 , and $70 \mathrm{mT}$. The transmission spectra for the corresponding perfectly ordered structures are also shown with solid lines. 


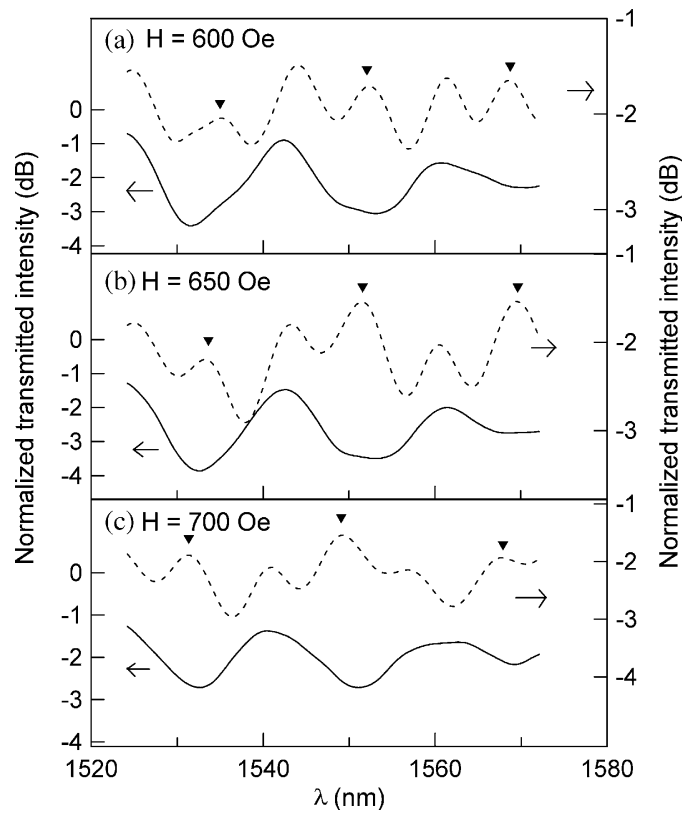

Fig. 5. Transmission spectra of the incident light through the ordered structures in the magnetic fluid film without (solid lines)/with (dashed lines) a point defect under various magnetic fields of (a) 60, (b) 65, and (c) $70 \mathrm{mT}$. The schemes of the incident and the transmitted directions of light through the magnetic fluid film are shown in Figs. 1(b) and (c). The corresponding field-dependent structural characters of the magnetic fluid film are shown in Figs. 3(a) and (b). The resonant wavelengths are labeled with triangles.

By comparing the dashed and the solid lines for a given field strength, the resonant wavelengths can be clearly recognized. They are labeled with tri-angles. The resonant wavelengths are shifted slightly to the short-wavelength region under a higher field. The slight shift in the resonant wavelength due to the varying magnetic field strength is repeatable and therefore reliable. Hence, it is evident that the resonant wavelength of the magnetic-fluid photonic crystal cavity can be manipulated by controlling the external magnetic field strength.

\section{Conclusion}

A self-assembly tunable ordered structure can be achieved in a magnetic fluid film under external magnetic fields. This ordered structure exhibits tunable photoniccrystal band gaps. When a point defect is artificially introduced into the ordered structure, the resonant modes can be observed. The resonant wavelength is also found to be magnetically tunable.

\section{Acknowledgment}

This work is supported by National Science Council of Taiwan under Grant nos. NSC94-2112-M-003-004,
93-2112-M-003-007, 94-2120-M-003-002，94-2112-M-003018, 94-2212-E-001, and by Ministry of Education, Taiwan under Grant no. 91-N-FA01-2-4-2.

\section{References}

[1] E. Yablonovitch, Phys. Rev. Lett. 58 (1987) 2059.

[2] S. John, Phys. Rev. Lett. 58 (1987) 2486.

[3] T. Yoshie, A. Scherer, H. Chen, D. Huffaker, D. Deppe, Appl. Phys. Lett. 79 (2001) 114.

[4] H.Y. Ryu, et al., Appl. Phys. Lett. 84 (2004) 1067.

[5] S.G. Johnson, J.D. Joannopoulos, Opt. Express 8 (2001) 173.

[6] C.-Y. Hong, I. Drikis, S.Y. Yang, H.E. Horng, H.C. Yang, J. Appl. Phys. 94 (2003) 2188.

[7] M. Notomi, Opt. Quant. Electron. 34 (2002) 133.

[8] S.Y. Yang, C.T. Chang, J. Appl. Phys. 98 (2005) 23108.

[9] E. Chow, S.Y. Lin, J.R. Wendt, S.G. Johnson, J.D. Joannopoulos, Opt. Lett. 26 (2001) 286.

[10] M. Koshiba, J. Lightwave Technol. 19 (2001) 1970.

[11] J.P. Berenger, J. Computat. Phys. 114 (1994) 185.

[12] H. Kosaka, T. Kawashima, A. Tomita, M. Notomi, T. Tamamura, T. Sato, S. Kawakami, Phys. Rev. B 58 (1998) R10096.

[13] X.-Y. Lei, H. Li, F. Ding, W. Zhang, N.-B. Ming, Appl. Phys. Lett. 71 (1997) 2889.

[14] T. Baba, Appl. Phys. Lett. 84 (2004) 4875.

[15] D.J. Norris, Y.A. Vlasov, Adv. Mater. 13 (2001) 371.

[16] H.B. Sun, S. Matsuo, H. Misawa, Appl. Phys. Lett. 74 (1999) 786.

[17] Z.-Y. Li, I. El-Kady, K.-M. Ho, S.Y. Lin, J.G. Fleming, J. Appl. Phys. 93 (2003) 38.

[18] T. Kawashima, et al., Appl. Phys. Lett. 77 (2000) 2613.

[19] P.K. Khizhenkov, Magn. Gidrodin 25 (1989) 21.

[20] P.K. Khizhenkov, V.L. Dorman, F.G. Bar'yakhtar, Magn. Gidrodin. 25 (1989) 35.

[21] A.O. Tsebers, Magn. Gidrodin 23 (1987) 15.

[22] A.O. Tsebers, Magn. Gidrodin 23 (1987) 59.

[23] M. Fermigier, A.P. Gast, J. Colloid Interf. Sci. 154 (1992) 522.

[24] J. Cernak, P. Macko, J. Magn. Magn. Mater. 123 (1993) 107.

[25] H. Wang, Y. Zhu, C. Boyd, W. Luo, A. Cebers, R.E. Rosensweig, Phys. Rev. Lett. 72 (1994) 1929.

[26] J. Liu, E.M. Lawrence, A. Wu, M.L. Ivey, G.A. Flores, K. Javier, J. Bibette, J. Richard, Phys. Rev. Lett. 74 (1995) 2828.

[27] C.-Y. Hong, I.J. Jang, H.E. Horng, C.J. Hsu, Y.D. Yao, H.C. Yang, J. Appl. Phys. 81 (1997) 4275.

[28] H.C. Yang, S.Y. Yang, W.S. Tse, Y.P. Chiu, H.E. Horng, C.-Y. Hong, Magn. Gidrodin. 35 (1999) 328.

[29] C.-Y. Hong, H.-E. Horng, F.C. Kuo, S.Y. Yang, H.C. Yang, J.M. Wu, Appl. Phys. Lett. 75 (1999) 2196.

[30] H.E. Horng, C.-Y. Hong, W.B. Yeung, H.C. Yang, Appl. Opt. 37 (1998) 2674.

[31] S.G. Johnson, J.D. Joannopoulos, Opt. Express 8 (2001) 173190.

[32] I. Drikis, S.Y. Yang, H.E. Horng, C.Y. Hong, H.C. Yang, J. Appl. Phys. 95 (2004) 5876.

[33] S.Y. Yang, I.J. Jang, H.E. Horng, C.-Y. Hong, H.C. Yang, Magnetohydrodynamics 36 (2000) 16.

[34] S.Y. Yang, Y.P. Chiu, H.E. Horng, C.-Y. Hong, B.Y. Jeang, H.C. Yang, Appl. Phys. Lett. 79 (2001) 2372.

[35] C.-Y. Hong, S.Y. Yang, H.E. Horng, H.C. Yang, J. Appl. Phys. 94 (2003) 3849. 